\title{
Balance of Monosynaptic Excitatory and Disynaptic Inhibitory Responses of the Globus Pallidus Induced after Stimulation of the Subthalamic Nucleus in the Monkey
}

\author{
Hitoshi Kita, ${ }^{1}$ Yoshihisa Tachibana, ${ }^{2}$ Atsushi Nambu, ${ }^{2}$ and Satomi Chiken ${ }^{1}$ \\ ${ }^{1}$ Department of Anatomy and Neurobiology, College of Medicine, University of Tennessee, Memphis, Tennessee 38163, and 2Division of System \\ Neurophysiology, National Institute for Physiological Sciences, and School of Life Science, The Graduate University for Advanced Studies, Myodaiji, \\ Okazaki 444-8585, Japan
}

\begin{abstract}
The subthalamic nucleus (STN) plays a pivotal role in controlling the activity of both the external and internal segments of the globus pallidus (GPe and GPi, respectively). Both nuclei receive monosynaptic excitatory and disynaptic GPe-mediated inhibitory inputs from the STN. Thus, we investigated the balance of these antagonistic inputs that may determine the overall response of pallidum to STN activation in monkeys. Single stimulation of the STN evoked a short-latency excitation followed by a weak inhibition in GPe neurons and a short-latency, very short-duration excitation followed by a strong inhibition in GPi neurons. Burst high-frequency stimulation (BHFS) (10 stimuli with $100 \mathrm{~Hz}$ ) of the STN (STN-BHFS) evoked powerful excitatory responses in GPe neurons. Local injection of a mixture of 1, 2, 3, 4-tetrahydro-6-nitro-2, 3-dioxobenzo[f]quinoxaline-7-sulfonamide (NBQX; AMPA/kainate receptor blocker) and 3-(2carboxypiperazin-4-yl)-propyl-1-phosphonic acid (CPP; NMDA receptor blocker) greatly diminished or abolished excitatory responses to the STN stimulation. In contrast to the GPe, STN-BHFS evoked a predominantly inhibitory response in GPi neurons. The inhibition could be blocked either by a local application of the $\mathrm{GABA}_{\mathrm{A}}$ receptor antagonist gabazine or by an injection of an NBQX/CPP/gabazine mixture into the GPe. STN-BHFS induced weak excitatory or inhibitory responses in a small number of phasically active putamen neurons. These data suggest that with single stimulation and during STN-BHFS, the STN-GPe excitatory response dominates over the STN-GPe-GPe recurrent inhibition in the GPe, whereas the STN-GPe-GPi inhibitory response dominates over the STN-GPi excitatory response in the GPi.
\end{abstract}

Key words: globus pallidus; subthalamic nucleus; unit recording; glutamatergic responses; GABAergic responses; awake monkey

\section{Introduction}

The subthalamic nucleus (STN) receives cortical, thalamic, and brainstem inputs and is considered to be an input nucleus of the basal ganglia (Kita, 1994). Stimulation of the motor cortex evokes a monosynaptic excitatory response that is overlapped by polysynaptic responses in the STN (Kitai and Deniau, 1981; Fujimoto and Kita 1993; Nambu et al., 2000). STN neurons respond very strongly to task-related events or sensorimotor stimuli (Matsumura et al., 1992; Bergman et al., 1994; Wichmann et al., 1994). The STN activity formed by the cortical and other inputs is conveyed mainly to the external segment of the globus pallidus (GPe) and the basal ganglia output nuclei, the internal segments of the globus pallidus (GPi), and the substantia nigra pars reticulata $(\mathrm{SNr})$ through branched axons (Kita et al., 1983b; Kita and

Received April 29, 2005; revised July 2, 2005; accepted Aug. 9, 2005

This work was supported by National Institutes of Health Grants NS-42762 and NS-47085 (H.K.), by Grants-in-Aid for Scientific Research on Priority Areas 17022042 from the Ministry of Education, Culture, Sports, Science and Technology of Japan (A.N.), and by the Naito Foundation (A.N.). We thank Dawn Merrick for editing this manuscript.

Correspondence should be addressed to Hitoshi Kita, Department of Anatomy and Neurobiology, College of Medicine, The University of Tennessee Memphis, 855 Monroe Avenue, Memphis, TN 38163. E-mail: hkita@utmem.edu.

D0I:10.1523/JNEUROSCI.1719-05.2005

Copyright $\odot 2005$ Society for Neuroscience $\quad$ 0270-6474/05/258611-09\$15.00/0
Kitai, 1987; Smith et al., 1990; Parent and Hazrati, 1995; Parent et al., 1999; Sato et al., 2000). The subthalamo-pallidal glutamatergic projections play pivotal roles in the control of the outputs of the basal ganglia and, hence, in motor control (Kitai and Kita, 1987; Bergman et al., 1990; Mink and Thach, 1993; Kita, 1994; Nambu, 2004). Pathological or experimental lesioning of the STN results in dyskinesia or hemiballismus (Dewey and Jankovic, 1989). In Parkinson's disease patients or animals, STN neurons often show higher firing rates and more burst activity than in normal subjects (Bergman et al., 1994; Hutchison et al., 1998). A lesion or high-frequency stimulation of the STN ameliorates some parkinsonian symptoms such as rigidity and akinesia (Bergman et al., 1990; Aziz et al., 1991; Benazzouz et al., 1993; Hutchison et al., 1998; Levy et al., 2000; Barlas et al., 2001).

The GPe is located in the strategic locus in the basal ganglia connections (Bergman et al., 1990; Mink and Thach, 1993; Kita, 1994; Nambu et al., 2000; Kita et al., 2004). The GPe receives its main inputs from the neostriatum and the STN and sends inhibitory projections mainly to the GPi/SNr and to the GPe itself through recurrent-collateral axons. Based on these connections, it can be expected that an activation of the STN should induce a monosynaptic excitation overlapped by a disynaptic inhibition in both the GPe and GPi. Thus, it can be assumed that the net 
outcome of the overlapped responses in the GPe and GPi to both single and high-frequency stimulation (HFS) of the STN might be critically dependent on the relative strength of these two opposing responses. To investigate this power balance issue, responses of pallidal neurons to single and burst high-frequency stimulation (BHFS) of the STN (STN-BHFS) were recorded in awake monkeys and were characterized by local application of glutamate and $\mathrm{GABA}_{\mathrm{A}}$ ionotropic receptor blockers. BHFS was used to mimic burst firing of STN neurons and also to address the effects of the continuous HFS of the STN, which has been used as a treatment for patients with Parkinson's disease (Benazzouz et al., 1996; Lopiano et al., 2001).

\section{Materials and Methods}

Monkey preparation. This study was performed in compliance with the guidelines of the National Institutes of Health Guide for Care and Use of Laboratory Animals and the National Institute of Physiological Sciences for the Use and Care of Laboratory Animals in Research. The monkey preparation methods used in the present study were very similar to those reported previously (Nambu et al., 2000; Kita et al., 2004). Macaca fuscata, named K11, K12, and K15 and a Macaca mulatta named K13 were trained to sit quietly in a monkey chair. The monkeys received a surgery to fix their heads painlessly to a stereotaxic frame attached to a chair. Under sodium pentobarbital ( $25 \mathrm{mg} / \mathrm{kg}$, i.v.) and ketamine hydrochloride $(10 \mathrm{mg} / \mathrm{kg}, \mathrm{i} . \mathrm{m}$.) anesthesia, the skull of the monkey was widely exposed, and two stainless-steel pipes were mounted in parallel over the frontal and occipital areas for head fixation. A few days after the surgery, the primary motor cortex (M1) and the supplementary motor area (SMA) were mapped by observing body part movements induced by cortical microstimulation and also by unitary responses to somatosensory stimuli (skin touch and passive joint movement) to identify the forelimb regions. After mapping, two pairs of stimulating electrodes (made of 200- $\mu$ m-diameter enamel-coated stainless-steel wire; intertip distance, $2 \mathrm{~mm}$ ) were implanted chronically in the forelimb region of the M1 and the SMA. To access the pallidum and STN, two holes $(10-15 \mathrm{~mm}$ diameter) were drilled in the skull. A rectangular shaped plastic chamber covering both holes, 30 and $55 \mathrm{~mm}$ long in the rostrocaudal and mediolateral directions, was fixed with an $\sim 20^{\circ}$ tilt from the horizontal plane onto the skull with acrylic resin.

Electrode assembly for unit recording and local injection of drugs. Recordings of pallidal units began after recovery from the surgery. During the experimental sessions, the monkeys were seated in a monkey chair with their heads restrained. Single-unit recordings of pallidal neurons in combination with local applications of $\mathrm{GABA}_{\mathrm{A}}$ or glutamate antagonists were performed with an electrode assembly consisting of a platinumiridium wire (catalog \#7675; A-M Systems, Carlsborg, WA) placed in a silica tube (outer diameter, $147 \mu \mathrm{m}$; inner diameter, $74 \mu \mathrm{m}$; part \#2000018; Polymicro Technologies, Phoenix, AZ) for unit recordings, two other silica tubes for drug delivery, and a protective stainless-steel tubing [for more details on the electrode assembly, see Kita et al. (2004)]. The impedance of the unit-recording electrode ranged from 0.7 to 1.2



The electrode assembly was penetrated obliquely $\left(45^{\circ}\right.$ from vertical) into the putamen (Put), GPe, and GPi. The syringes contained the AMPA/kainate blocker 1, 2, 3, 4-tetrahydro-6-nitro-2, 3-dioxobenzo[f] quinoxaline-7-sulfonamide (NBQX; 0.5-1 mM), the NMDA antagonist 3-(2-carboxypiperazin-4-yl)-propyl-1-phosphonic acid (CPP; $0.5-1 \mathrm{~mm}$ ), or the $\mathrm{GABA}_{\mathrm{A}}$ antagonist gabazine (0.2-1 mM) dissolved in saline. A total volume of $0.1-0.2 \mu \mathrm{l}$ was injected at a rate of $0.03 \mu \mathrm{l} / \mathrm{min}$ (i.e., the total injection time was 3-7 $\mathrm{min}$ ) by advancing the plungers with computer-controlled stepping motor driven actuators. Our previous study, using the same injection method, indicated that the drug effect became maximum $5-10$ min after the injection and the radius of the effective area was $<1 \mathrm{~mm}$ with a $0.2 \mu \mathrm{l}$ injection (Kita et al., 2004). Thus, once an injection was made, the next injection site was separated by $\geq 1$ $\mathrm{mm}$ from the previous injection site. For chemical activation of the STN, the same electrode assembly was penetrated vertically into the STN, and gabazine (0.2 mM; 0.4-1.0 $\mu \mathrm{l})$ was injected.

\section{A: STN single stim. $0.7 \mathrm{~mA}$}

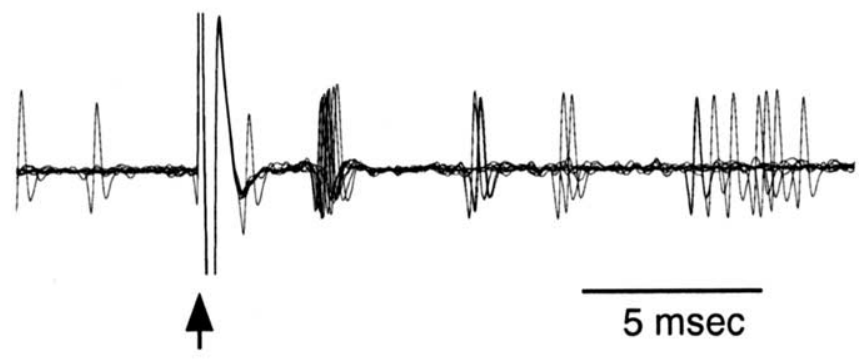

B: STN double stim. $0.7 \mathrm{~mA}$

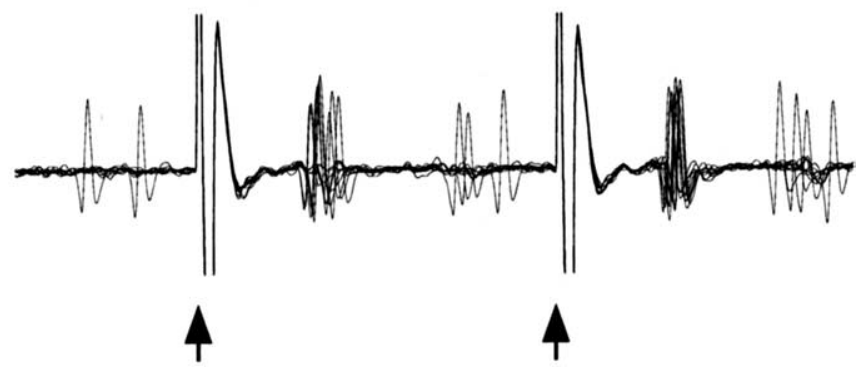

Figure 1. $\boldsymbol{A}, \boldsymbol{B}$, Responses of a GPe neuron to single stimulation (stim.; $\boldsymbol{A}$ ) and double stimulation (stim.; $\boldsymbol{B}$ ) of the STN. Ten overlapped traces show that single or double stimulation of the STN (marked by arrows) induced highly synchronized spiking $\sim 3.5 \mathrm{~ms}$ after each stimulus. The second spikes following the initial short-latency spikes were also synchronized. Stimulus artifacts lasted $\sim 1 \mathrm{~ms}$ and hampered the detection of the spikes that occurred during those periods.

Unit recordings and data analysis. The STN was stimulated by a concentric-bipolar electrode with a stainless-steel center lead (diameter, $120 \mu \mathrm{m}$ ) and a stainless-steel outer tube (outer diameter, $0.35 \mathrm{~mm}$ ), with a tip separation of $0.5 \mathrm{~mm}$ and an impedance of $\sim 5 \mathrm{~K} \Omega$ (measured with $1 \mathrm{kHz} \mathrm{AC}$ ). To place the electrode in the forelimb regions of the STN, multiunit spontaneous activity and responses to stimulation of the M1 and SMA were recorded through the center lead of the stimulus electrode. The forelimb regions of the STN were identified as having neurons with $\sim 25 \mathrm{~Hz}$ irregular spontaneous firing and a specific STN response pattern consisting of a short-latency excitation following an inhibition and a slow excitation to cortical stimulation, as reported previously (data not shown) (Matsumura et al., 1992; Bergman et al., 1994; Wichmann et al., 1994; Nambu et al., 2000). The duration of the stimulus current pulses was always $300 \mu \mathrm{s}$. Other stimulus parameters were as follows: single stimulation, strength up to $0.7 \mathrm{~mA}$, delivered every $1.4 \mathrm{~s}$; double stimuli, strength up to $0.7 \mathrm{~mA}$, delivered every $2.4 \mathrm{~s}$; BHFS with 10 burst $(100 \mathrm{~Hz})$ pulses, strength up to $0.4 \mathrm{~mA}$, usually delivered every $5.4 \mathrm{~s}$ unless otherwise indicated; and BHFS with 20 burst $(100 \mathrm{~Hz})$ pulses, strength up to $0.4 \mathrm{~mA}$, delivered every $10.4 \mathrm{~s}$. Stimulation with these parameters did not induce any body movements.

The unit recordings were amplified and passed through a $0.7-2 \mathrm{kHz}$ bandpass filter and then to a homemade window discriminator that was capable of excluding stimulus artifacts. When the strongest intensity stimulus used in this study, $0.7 \mathrm{~mA}$, was applied to the $\mathrm{STN}$, a stimulus artifact lasted $\sim 1 \mathrm{~ms}$, and any firing that occurred during this period was undetectable (Fig. 1). The artifacts created short, one or two bins, gaps, or shorting of bins in the peristimulus time histograms (PSTHs) immediately after the stimulation. The artifacts might lead to $10 \%$ underestimation of firing rate during $100 \mathrm{~Hz}$ BHFS, when an even distribution of spikes is assumed. Stimulation of the STN or the GPi resulted in a sharp excitation of the GPe and GPi neurons $4-5 \mathrm{~ms}$ after stimulation (Nambu et al., 2000; Hashimoto et al., 2003; Bar-Gad et al., 2004). Because the main excitation peaks are detectable in $100 \mathrm{~Hz}$ BHFS (see Fig. 3), we 
A-a: Control, single stim. $0.7 \mathrm{~mA}$

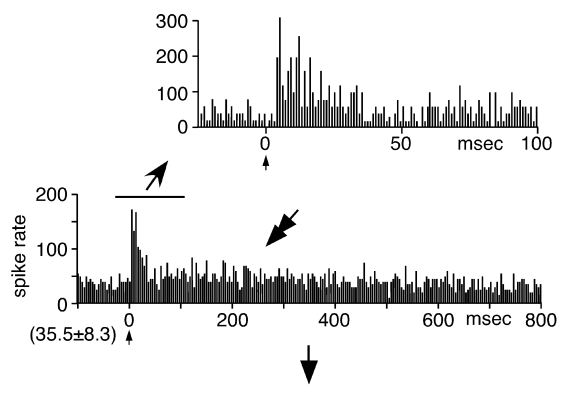

A-b: After local NBQX/CPP mixture

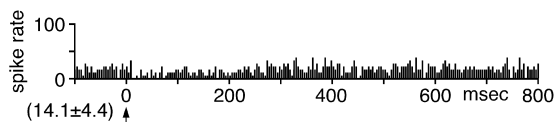

B-a: Control, single stim. $0.7 \mathrm{~mA}$

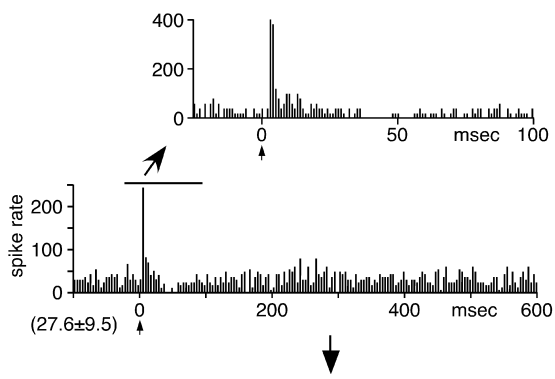

B-b: After local NBQX/CPP/gabazine mixture



Figure 2. Responses of two GPe neurons $(\boldsymbol{A}, \boldsymbol{B})$ to single stimulation (stim.) of the STN. $\boldsymbol{A}-\boldsymbol{a}$, A neuron responded with a short-latency excitation followed by a small and very slow excitation (marked by a double arrow). $\boldsymbol{A}-\boldsymbol{b}$, To block STN-GPe excitation, a mixture of ionotropic glutamate blockers (NBQX and CPP, $0.5 \mathrm{~mm}$ each; $0.2 \mu \mathrm{l}$ ) was locally applied. The blockers greatly decreased the rate of spontaneous firing and suppressed both the short-latency and slow excitations. $\boldsymbol{B}$ - $\boldsymbol{a}$, This neuron evoked a short-latency, short-duration excitation followed by an inhibition. $\boldsymbol{B}-\boldsymbol{b}$, Local application of a mixture of ionotropic glutamate and $\mathrm{GABA}_{\mathrm{A}}$ blockers (NBQX, CPP, and gabazine, $0.5 \mathrm{~mm}$ each; $0.2 \mu \mathrm{l}$ ) blocked short-latency excitation and inhibition without a large reduction of the spontaneous firing rate. In $\boldsymbol{A}$ and $\boldsymbol{B}$, the PSTHs with the fast and slow time scale were constructed with 1 and $4 \mathrm{~ms}$ bins, respectively. In this and the following figures, the mean \pm SD of prestimulus firing frequency is indicated in the parentheses located at the bottom left of each PSTH. Also, one or two bin-wide gaps or low bins in some PSTHs immediately after stimulus are attributable to stimulus artifacts (see Materials and Methods for more details).

considered that the actual error would not significantly change the results presented in our study.

The responses of pallidal neurons to the STN stimulation were assessed by constructing PSTHs for 50 single-stimulation trials and 25 burst-stimulation trials. The ordinate of all of the PSTHs was converted to the firing rate. PSTHs with $1 \mathrm{~ms}$ bin width were constructed on-line for initial evaluations and, in some neurons, PSTHs with $4 \mathrm{~ms}$ bin width were used in the off-line analysis. Mean values and SDs of the firing rate during the $100 \mathrm{~ms}$ preceding the stimulation were calculated from PSTHs and were considered to be the values for base discharge. The changes in the firing activity in response to STN stimulation were judged to be significant if the firing rate during at least two consecutive bins of PSTHs reached the statistical level of $p<0.05$ (one-tailed $t$ test). The amplitudes and durations of slow responses to STN stimulation were obtained from PSTHs with $4 \mathrm{~ms}$ bins. The changes in unitary responses by local injections of glutamate and $\mathrm{GABA}_{\mathrm{A}}$ antagonists were assessed by measuring the peak response amplitude, an average of the two highest for excitation or of the two lowest for inhibition from the mean prestimulus firing level. Because the drug effects became strongest 5-10 min after injection and decayed very slowly (Kita et al., 2004), the spontaneous firing rate, amplitudes, and durations of the responses to STN stimulation obtained before and 5-10 min after drug injection were evaluated by a paired $t$ test.

Several of the recording and drug injection sites were marked by passing a cathodal direct current $(20 \mu \mathrm{A}$ for $30 \mathrm{~s})$ through the recording electrode. At the end of the final experiment, the monkeys were killed, and the recording sites were reconstructed from histological sections of the brains according to the lesions made by current injections and by the traces of the electrode tracks, using the methods described previously (Nambu et al., 2000) (data not shown).

\section{Results}

\section{Responses of GPe neurons to STN stimulation}

The neurons included in the present study were the highfrequency firing with pause type of GPe neurons, which is the most numerous type in monkey GPe (DeLong 1971). A total of 37 GPe neurons that responded to the stimulation of the STN were included in this report. These neurons were also tested by stimulation of the motor cortices, and it was confirmed that these neurons were in the forelimb regions of the GPe (data not shown) (Nambu et al., 2000; Kita et al., 2004). These neurons were obtained from monkeys $\mathrm{K} 11(n=4)$, $\mathrm{K} 12(n=5), \mathrm{K} 15(n=11)$, and $\mathrm{K} 13(n=$ 14 ). The neurons were pooled as the spontaneous firing and responses to STN stimulation obtained from the four monkeys were very similar.

The mean spontaneous firing rate of GPe neurons was $64.1 \pm 24.4 \mathrm{~Hz}(n=37)$. Short, constant, latency spikes capable of following double stimulation with $>200$ $\mathrm{Hz}$, suggestive of antidromic responses, were observed in 3 of 37 neurons examined (data not shown). In the 34 neurons examined for orthodromic responses, single STN stimulation evoked either a shortlatency excitation $(n=16)$ (Fig. $2 A)$, a short-latency excitation followed by a weak inhibition $(n=15)$ (Fig. $2 B)$, or a weak inhibition only $(n=3$; data not shown) in GPe neurons. In some neurons, STN stimulation reset the timing of the firing (Fig. 1) and created very narrow and high peaks and depressions in the excitatory response portion of PSTHs (Fig. $2 B$ a). In approximately half of the excitationonly neurons, the inhibitions following the excitation could be visually recognized in the PSTH but failed to reach a statistically significant level. The latency and the duration of the excitation was $4.2 \pm 1.2$ and $20.0 \pm 7.3 \mathrm{~ms}$, respectively $(n=31)$. The duration of the excitation tended to be shorter when it was followed by an inhibition, an average $25.7 \pm 8.2 \mathrm{~ms}$ without inhibition versus $11.0 \pm 3.5 \mathrm{~ms}$ with inhibition $(p<0.05)$. The latency and the duration of the inhibitions were $15.3 \pm 8.2$ and $68.5 \pm$ $46.5 \mathrm{~ms}$, respectively $(n=15)$. In eight GPe neurons, single-STN stimulation evoked a small and very slow excitation in addition to the short-latency responses (Fig. $2 A-a$ ). Local injection of ionotropic glutamate blockers (a mixture of $0.5 \mathrm{~mm} \mathrm{NBQX}$ and 0.5 $\mathrm{mM}$ CPP) greatly diminished or abolished both the short-latency excitations and inhibitions and also the slow excitations to STN stimulation (Fig. $2 A-b$ ). The NBQX/CPP mixture also greatly decreased the rate of spontaneous firing $(58.4 \pm 28.8$ to $17.4 \pm$ $8.5 ; n=6 ; p<0.002$ ) as reported previously (Kita et al., 2004). Local application of ionotropic glutamate and $\mathrm{GABA}_{\mathrm{A}}$ blockers (a mixture of $0.5 \mathrm{~mm} \mathrm{NBQX,} \mathrm{0.5} \mathrm{mM} \mathrm{CPP,} \mathrm{and} 0.5 \mathrm{~mm}$ gabazine) blocked both the short-latency excitation and the following inhibition with no significant change in the level of spontaneous firing ( $62.2 \pm 29.8$ to $68.4 \pm 28.5 ; n=5 ; p>0.05$ ) (Fig. $2 B-b$ ) (Kita et al., 2004). The NBQX/CPP/gabazine mixture became a useful tool in a later experiment.

Thirty-one of the 34 GPe neurons examined with single stimulation were also tested with STN-BHFS. During stimulation, 28 neurons evoked a series of short-latency excitations, and the average firing rate increased. The other three neurons failed to evoke significant responses. The termination of the BHFS was followed by either a small inhibition followed by a slow excitation $(n=16)$ (Fig. 3$)$ or by a slow excitation only $(n=12)$ (Fig. 4$)$. During the BHFS, two forms of potentiations of short-latency excitations were often observed. During BHFS, the excitation to each stimulation showed progressive potentiation (Fig. $3 B-D$ ). The waxing appeared to reach a steady level by the 5 th to 10 th 
stimulation (Fig. 3D). The potentiated excitation was maintained for the duration of the longer periods of BHFS (Fig. 3). Another change was a decrease in the stimulus threshold, a phenomenon similar to the posttetanic potentiation. As a result of this change, the threshold stimulus current for the BHFS was much lower than that for the single stimulation. For example, Figure 3 shows responses of a GPe neuron to single and STN-BHFS. Single stimulation with $0.4 \mathrm{~mA}$ evoked an insignificant responses in this GPe neuron (Fig. 3A). BHFS with 0.4 $\mathrm{mA}$ evoked strong short-latency excitations even to the first of the burst stimulus pulses (Fig. 3B). This potentiation was further tested in three neurons by using longer interburst intervals. The potentiation was observed with all 20.4, 10.4, and standard $5.4 \mathrm{~s}$ interburst intervals (data not shown). Stimulation with lower currents decreased the magnitudes of all of the response components. Specifically, the slow inhibition was no longer observed, and the peak latency of the slow excitation shortened as the stimulus intensity decreased (Fig. 3, compare $B$ and $C$ ).

Local application of an NBQX/CPP mixture greatly suppressed all of the response components evoked after STNBHFS. The glutamate blockers also greatly decreased the rate of spontaneous firing as mentioned previously (Fig. 4A). Local application of a mixture of NBQX, CPP, and gabazine also greatly suppressed all of the responses without significantly changing the spontaneous firing rate, as reported previously $(n=7)$ (Fig. 4B).

\section{Responses of GPi neurons to STN stimulation}

A total of 29 GPi neurons responsive to STN stimulation were recorded from monkeys $\mathrm{K} 11(n=3), \mathrm{K} 12(n=4), \mathrm{K} 15(n=$ $10)$, and $\mathrm{K} 13(n=12)$. These neurons also responded to stimulation of the M1 or the SMA (data not shown) (Nambu et al., 2000). The neurons were pooled, because the spontaneous firing and responses to STN stimulation obtained from the four monkeys were very similar. All of the neurons had continuous firing with the mean rate of $58.5 \pm 16.8 \mathrm{~Hz}(n=29)$. Single stimulation of the STN evoked a shortlatency and a short-duration excitation followed by a strong inhibition in 21 of 29 GPi neurons (Fig. 5). Five neurons evoked a short-latency excitation followed by a small inhibition that was visually recognizable in the PSTH but statistically insignificant, and three others evoked an inhibition only (data not shown). None of the GPi neurons evoked short and constant latency antidromic-like spikes to STN stimulation. STN stimulation reset the timing of firing of some GPi neurons, as reported previously (Hashimoto et al., 2003), and created very narrow and high peaks and depressions in the excitatory response portion of PSTHs

B: burst stim. $0.4 \mathrm{~mA}$ respectively.


D: longer burst stim. $0.4 \mathrm{~mA}$

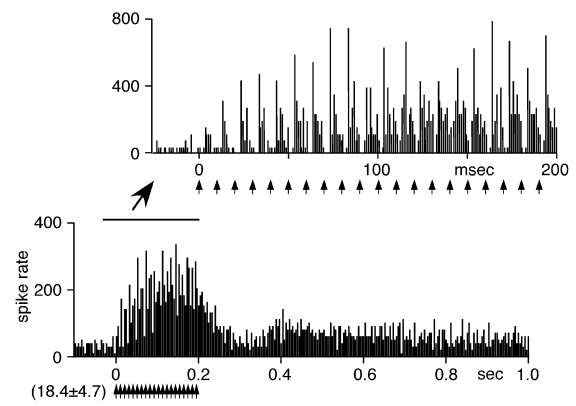

Figure 3. STN-BHFS evoked excitations in a GPe neuron. $\boldsymbol{A}$, The threshold intensity for single STN stimulation (stim.) was $\sim 0.4 \mathrm{~mA}$. B, STN-BHFS with $0.4 \mathrm{~mA}$ (10 pulses with $100 \mathrm{~Hz}$ ) evoked short-latency excitations during stimulation that were followed by a small inhibition and another long-lasting slow excitation after the termination of the stimulation. During BHFS, the short-latency excitation to each stimulation showed progressive potentiation (waxing). The excitation to the first of 10 burst stimulus pulses was always stronger than that evoked by a single stimulation with the same intensity (compare $\boldsymbol{A}$ and $\boldsymbol{B}$ ). $\boldsymbol{C}$, Lower current intensity $(0.1 \mathrm{~mA})$ BHFS was still able to induce excitatory responses. $\boldsymbol{D}$, Responses to BHFS with twice the duration $(0.4 \mathrm{~mA} ; 20$ pulses with $100 \mathrm{~Hz})$. The waxing of the short-latency excitations reached the maximum level at approximately the 10th stimulus. PSTHs with fast ( -25 to $200 \mathrm{~ms}$ ) and slow ( -0.1 to $1.2 \mathrm{~s}$ ) time scales were constructed with 1 and 4 ms bins,

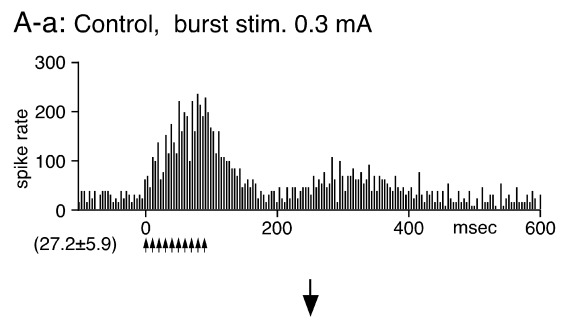

A-b: After local NBQX/CPP mixture

B-a: Control, burst stim. $0.2 \mathrm{~mA}$

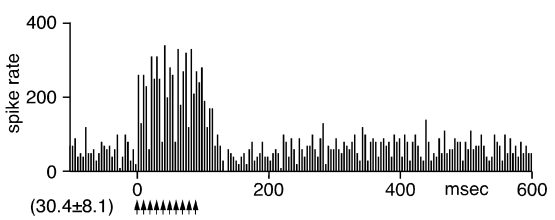

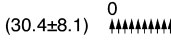

\section{$\downarrow$}

B-b: After local NBQX/CPP/gabazine mixture
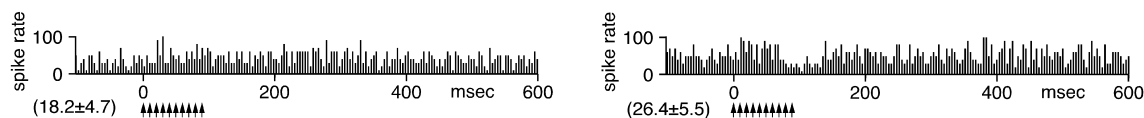

Figure 4. Effects of local application of a mixture of NBQX and CPP and a mixture of NBQX, CPP, and gabazine on GPe neurons. Recordings in $\boldsymbol{A}$ and $\boldsymbol{B}$ are from two different neurons. $\boldsymbol{A}-\boldsymbol{a}$, In the control, STN-BHFS evoked short-latency excitations during stimulation (stim.) and a slow excitation after the termination of the stimulation. $\boldsymbol{A}-\boldsymbol{b}$, Local application of a mixture of NBQX and CPP (0.5 mm each, $0.2 \mu \mathrm{l})$ greatly suppressed both the short-latency and the slow excitations. The glutamate blockers also greatly decreased the rate of spontaneous firing. $\boldsymbol{B}-\boldsymbol{a}$, In the control, STN-BHFS evoked a short-latency excitation during stimulation (stim.) and a weak, slow excitation after the stimulation in this GPe neuron. $\boldsymbol{B}$ - $\boldsymbol{b}$, Local application of a mixture of NBQX, CPP, and gabazine $(0.5 \mathrm{~mm}$ each; $0.2 \mu \mathrm{l})$ greatly suppressed the excitations without causing large changes in the spontaneous firing rate. All of the PSTHs were constructed with 4 ms bins.

(Figs. 5, 7A). In 9 of the 21 neurons that evoked excitationinhibition, the inhibition abruptly ended and was followed by a rebound-like short excitation (Fig. 5B). The mean latency of the short-latency excitation was $3.9 \pm 0.4 \mathrm{~ms}$, which was $\sim 0.4 \mathrm{~ms}$ shorter than that of the GPe neurons. The mean duration of the short-latency excitation was $9.3 \pm 5.8 \mathrm{~ms}$ and was significantly shorter than that of the GPe neurons $(p<0.01)$. The latency and duration of the inhibition was $13.9 \pm 4.2$ and $40.3 \pm 15.2 \mathrm{~ms}$, respectively. Local application of gabazine abolished the inhibition and increased the rate of spontaneous firing (Fig. 5). The 
A-a: Control, single stim $0.7 \mathrm{~mA}$

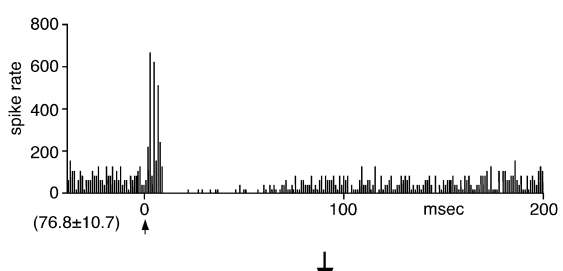

A-b: After local gabazine

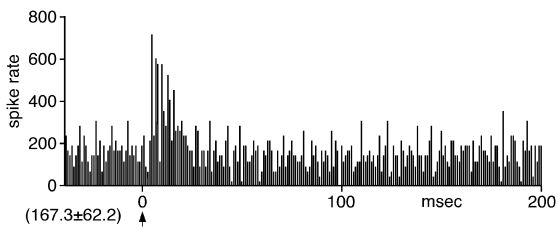

B-a: Control, single stim $0.5 \mathrm{~mA}$


Figure 5. Single stimulation of the STN evoked a short-latency and a very short-duration excitation, followed by an inhibition in GPi neurons. Recordings in $\boldsymbol{A}$ and $\boldsymbol{B}$ are from two different neurons. In some neurons, the STN stimulation (stim)-induced inhibition was followed by a rebound-like excitation $(\boldsymbol{B}-\boldsymbol{a})$. Local application of the $G A B A_{A}$ blocker gabazine greatly decreased the inhibition and also increased the rate of spontaneous firing $(\boldsymbol{A}-\boldsymbol{b}, \boldsymbol{B}-\boldsymbol{b})$. Gabazine also blocked the rebound-like excitation $(\boldsymbol{B}$ - $\boldsymbol{b})$. The short-latency excitation observed after local gabazine application was very similar to that observed in the GPe. All of the PSTHs were constructed with $1 \mathrm{~ms}$ bins.

A-a: Control, burst stim. $0.2 \mathrm{~mA}$

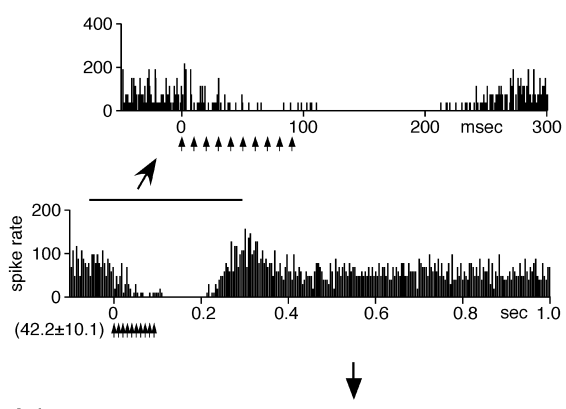

A-b: After local gabazine

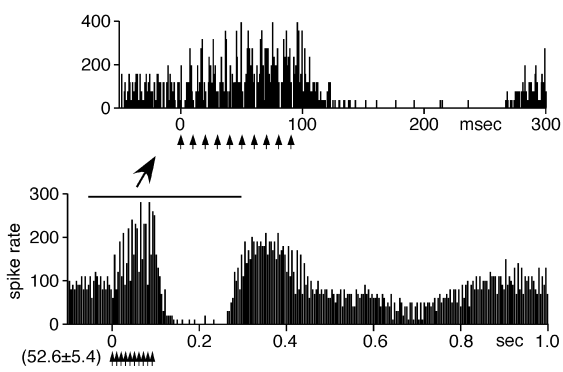

B-a: Control, burst stim. $0.2 \mathrm{~mA}$

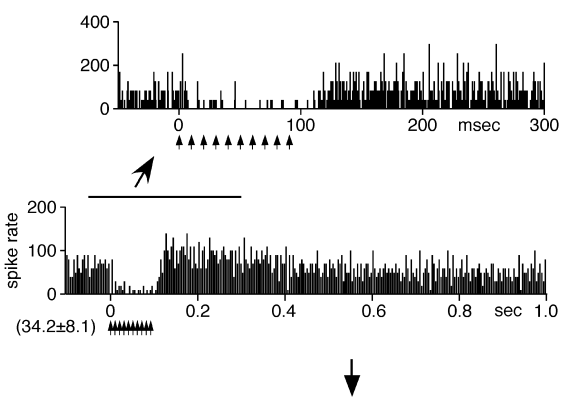

B-b: After local gabazine



Figure 6. STN-BHFS evoked a long inhibition in a majority of GPi neurons. Recordings in $A$ and $\boldsymbol{B}$ are from two different neurons. $\boldsymbol{A}-\boldsymbol{a}, \boldsymbol{B}-\boldsymbol{a}$, STN-BHFS with $0.2 \mathrm{~mA}$ evoked a brief, small excitation followed by an inhibition in these neurons. The inhibition evoked in neuron A lasted for $\sim 120$ ms after the termination of the stimulation (stim.), whereas in neuron B, the inhibition ended shortly after the termination of the stimulation (stim.). The inhibition often terminated abruptly and, in approximately one-half of the GPi neurons, was followed by a slow, long-lasting excitation. $\boldsymbol{A}-\boldsymbol{b}, \boldsymbol{B}-\boldsymbol{b}$, Local application of gabazine greatly suppressed the inhibition during burst stimulation. However, gabazine was less effective during the later part of the inhibition that occurred after the termination of the stimulation. Gabazine also increased the rate of spontaneous firing and the amplitude of the excitation after the inhibition. PSTHs with fast ( -50 to $300 \mathrm{~ms})$ and slow $(-0.1$ to $1.0 \mathrm{~s})$ time scales were constructed with 1 and 4 ms bins, respectively.

duration of the short-latency excitation was increased to $45 \pm$ $12.8 \mathrm{~ms}(n=5 ; p<0.01)$. Gabazine also abolished the reboundlike excitation (Fig. $5 B$ ).

Twenty-four of the 29 GPi neurons examined with single stimulation were also tested with STN-BHFS. In 18 neurons,
BHFS evoked a brief 3-16 ms shortlatency excitation at the beginning of the BHFS, followed by an inhibition. The inhibition lasted for various durations, from 100 to $300 \mathrm{~ms}$ after the termination of the BHFS, was often abruptly terminated, and was followed by an excitation (Fig. 6). Local application of gabazine blocked the inhibition during BHFS and disclosed excitations. However, gabazine was less effective during the later part of the inhibition that occurred after the termination of the BHFS. Gabazine also increased the rate of spontaneous firing and the amplitude of the excitation following the inhibition (Fig. 6). The six remaining neurons evoked a series of excitation during stimulation followed by an inhibition with the response pattern very similar to GPe neurons (data not shown).

To block disynaptic STN/GPe/GPi inputs at the GPe and to maintain the spontaneous firing level of the GPe, a mixture of NBQX, CPP, and gabazine $(0.5 \mathrm{~mm}$ each; $0.4-0.6 \mu \mathrm{l}$ ) was injected into the GPe 5-30 min before GPi recording. The injection was made while the electrode assembly for GPi recording was passing through the GPe at approximately the middle of the tract length of the nucleus. After the injection, both single and BHFS of the STN did not evoke inhibition in any of the eight GPi neurons tested but did evoke predominantly short-latency excitations (Fig. 7). The slow excitation observed before GPe blockade was also not observed in any of the eight neurons (Fig. 7). We tested a mixture of NBQX and CPP $(0.5 \mathrm{~mm}$ each; $0.4-0.6 \mu \mathrm{l})$ in the same experimental paradigm. Injections of this mixture into the GPe greatly increased the spontaneous firing level of the GPi (55.7 \pm 25.3 to $102.3 \pm 28.2 \mathrm{~Hz} ; n=6 ; p<0.01$ ) and obscured the excitatory responses (data not shown).

\section{Responses of pallidal neurons to} chemical activation of the STN

In addition to the electrical stimulation results, we examined the effect of gabazine injections into the STN on the level of the spontaneous firing of GPe and GPi neurons. It was expected that a blockade of the GABAergic pallidal inputs to the STN should increase the spontaneous activity of STN neurons (Kita et al., 1983a). Indeed, local injection of gabazine $(0.2 \mathrm{~mm}$; $0.4-1.0 \mu \mathrm{l}$ ) greatly increased spontaneous activity of STN neurons with a decrease of the amplitude of spikes, although a precise measurement of the increase was not possible, because our electrode assembly permitted only multiunit recordings (data not shown). The average firing frequencies of GPe and GPi neurons were obtained from $50 \mathrm{~s}$ of sampling 


\section{A: After application of NBQX/CPP/gabazine in the GPe single stim. $0.6 \mathrm{~mA}$}



\section{B: After application of NBQX/CPP/gabazine in the GPe burst stim. $0.2 \mathrm{~mA}$}

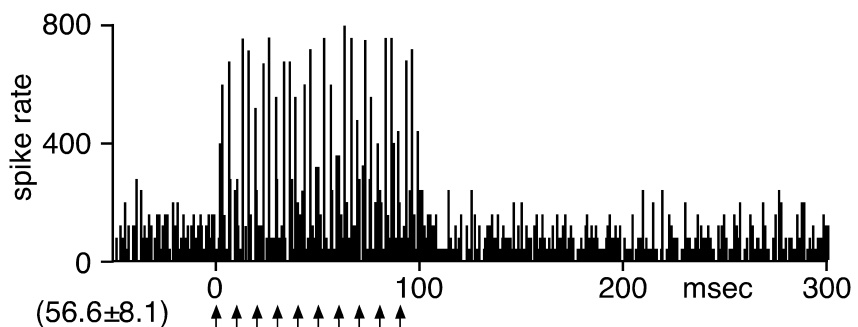

\section{C: After local NBQX/CPP mixture}

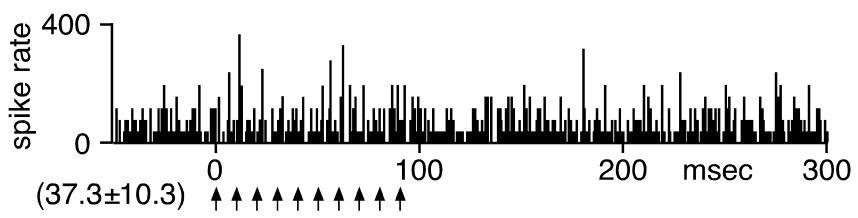

Figure 7. The blockade of the GPe suppressed the inhibition of GPi neurons evoked by STN stimulation. Before GPi recording, a mixture of NBQX, CPP, and gabazine ( $0.5 \mathrm{~mm}$ each; $0.6 \mu \mathrm{l}$ ) was injected into the GPe to block STN stimulation-induced responses in that nucleus. $\boldsymbol{A}$, Single stimulation (stim.) of the STN evoked a short-duration, short-latency excitation. $\boldsymbol{B}$, STN-BHFS evoked short-latency excitations. stim., Stimulation. C, Local application of the NBQX/CPP mixture greatly suppressed the excitation during BHFS. The PSTH in $\boldsymbol{A}$ was constructed with $1 \mathrm{~ms}$ bins, and those in $\boldsymbol{B}$ and $\boldsymbol{C}$ were constructed with $4 \mathrm{~ms}$ bins.

times before and after the gabazine injection into the STN. The numbers of neurons tested were small, because the gabazine injection can be done only once or twice a day in monkeys. Six GPe neurons all increased the firing rate after the gabazine injection into the STN $(47.0 \pm 19.5$ to $96.8 \pm 35.0 \mathrm{~Hz} ; p<0.02)$. The firing increase lasted for $\geq 1 \mathrm{~h}$ with the peak effect at 5-15 min after the injection. Of 10 GPi neurons tested, 4 increased and 6 decreased (Fig. 8), and as a group, there was no significant change in the firing rate, the mean firing rate of $57.5 \pm 9.1$ before the injection to $57.6 \pm 30.6 \mathrm{~Hz}$ after.

Gabazine injection into the STN occasionally induced dyskinetic movements of the contralateral forelimb. The dyskinesia was mild and was not so evident as in the muscimol injection (Nambu et al., 2000). Raisin presenting tests also revealed slow and disturbed precision grip. When removed from the chair soon after the injection, the monkeys occasionally showed a body tilt and a circling behavior to the contralateral side to the injection site.

\section{Responses of Put neurons to STN stimulation}

It is possible that stimulation of the STN antidromically activates the cerebral cortex or the centromedial thalamic nucleus and that these activations then orthodromically activate striatopallidal
A

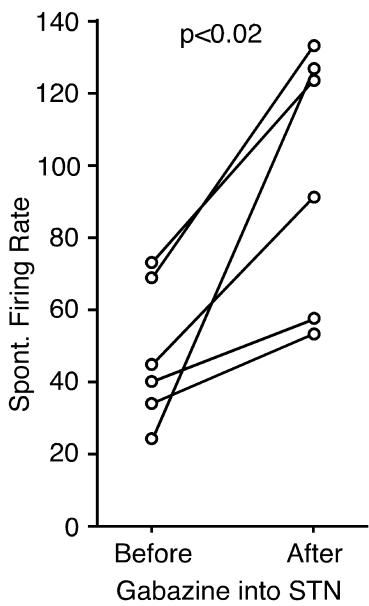

B $\quad$ GPi

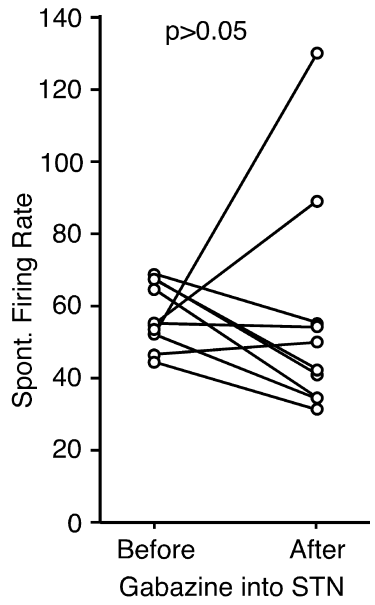

Figure 8. Effects of gabazine injection $(0.2 \mathrm{~mm} ; 0.4-0.6 \mu \mathrm{l})$ into the STN on the spontaneous (Spont.) firing level of six GPe $(\boldsymbol{A})$ and $10 \mathrm{GPi}(\boldsymbol{B})$ neurons.

neurons. To test this possibility, 22 spontaneously inactive Put neurons with evoked excitation to cortical stimulations that we considered phasically active neurons (PANs) and 28 tonically active neurons (TANs) were tested by single and BHFS of the STN. PANs and TANs were identified by their firing frequency, firing patterns, and spike width (Crutcher and DeLong, 1984; Kimura et al., 1984, 1990). Neurons were sampled from the area of the Put that exhibited orthodromic responses to the stimulation of the forelimb regions of the motor cortex. Single stimulation of the STN failed to activate any PANs. STN-BHFS induced slowly growing, weak excitation in three PANs (Fig. 9A). In 22 TANs, single STN stimulation induced an excitation with a 20-30 ms latency followed by a weak inhibition-excitation sequence (Fig. 9B-a). However, when these neurons were tested with STN-BHFS, the excitations and inhibitions appeared to cancel each other, and no clear response could be evoked (Fig. 9B-b).

\section{Discussion}

\section{Stimulus method}

The effectiveness and the types of neuronal elements stimulated by an electrical field are dependent on the method of stimulation. We used a vertical penetration of a concentric bipolar electrode with small contact areas separating $0.5 \mathrm{~mm}$, which should produce a strong voltage gradient in a small area around the center lead. We considered that the stimulus method was inefficient in stimulating axons, because only a small number of GPe neurons and none of GPi neurons, the axons of which pass near the STN (Parent et al., 1999), evoked an antidromic response. For the same reason, we suspect that strong stimulation of smalldiameter dopamine and serotonin axons to be unlikely. The electrodes for the STN-HFS in clinical use are typically penetrated in the rostrodorsal to caudoventral direction. The electrodes have large contact areas with long contact separations that effectively stimulate large myelinated axons, including axons of STN neurons, oriented parallel to the electrode (McIntyre et al., 2004).

\section{Short-latency responses}

The present results revealed that responses of GPe and GPi neurons to STN stimulation differ from each other, although both nuclei receive anatomically similar monosynaptic excitatory and 


\section{A: PAN, burst stim. $0.4 \mathrm{~mA}$}

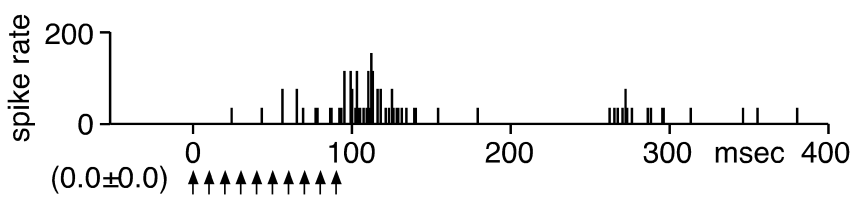

B-a: TAN, single stim. $0.7 \mathrm{~mA}$

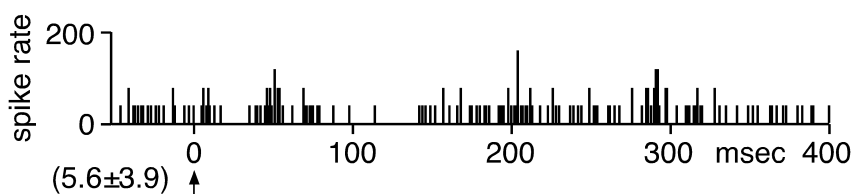

B-b: TAN, burst stim. $0.4 \mathrm{~mA}$

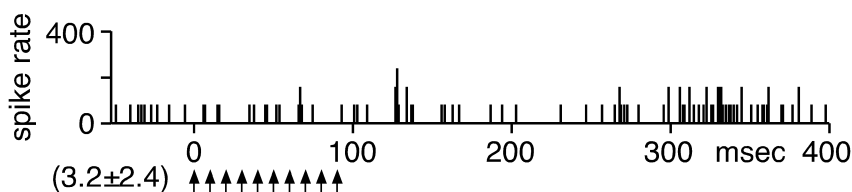

Figure 9. Responses of Put neurons to STN stimulation (stim.). $\boldsymbol{A}$, STN-BHFS induced a weak excitation in a PAN. $\boldsymbol{B}-\boldsymbol{a}$, Single STN stimulation evoked an excitation with a $20-30$ ms latency followed by a weak inhibition-excitation sequence in a TAN. $\boldsymbol{B}$ - $\boldsymbol{b}$, However, when the same neuron was tested with STN-BHFS, no significant response was evoked.

disynaptic inhibitory connections. The primary difference was the strength of the disynaptic STN-GPe-pallidal inhibition. GPe neurons activated by STN stimulation can induce stronger inhibition in the GPi than in the GPe. This result is consistent with the anatomical finding that there is a higher density of GPe synapses on GPi neurons than GPe neurons (Shink and Smith, 1995). In the GPe, in both single and BHFS of the STN, the STN-GPe excitation dominated over the STN-GPe-GPe recurrent inhibition. As expected, local application of the NBQX/CPP mixture diminished the short-latency excitations and the subsequent inhibition in the GPe (Kita and Kitai, 1991; Kita et al., 2004), suggesting that the inhibition was the recurrent collateral inhibition and not a result of antidromic activation of GPe-STN axons.

Short-latency excitations to STN-BHFS showed two forms of potentiation. One was the waxing of succeeding excitations to burst stimulations. This potentiation could be attributable to a property of the STN-GPe synapses, because unitary EPSPs recorded in rat pallidal neurons in slice preparations showed similar waxing (Hanson and Jaeger, 2002). The other form of potentiation was similar to posttetanic potentiation in that repetitive BHFS potentiated responses to succeeding BHFS. This potentiation was observed even in $20.4 \mathrm{~s}$ interburst intervals, suggesting the involvement of slow mechanisms.

In the GPi, the most frequently encountered response pattern to single stimulation of the STN was a short-latency excitation followed by an inhibition. The duration of the excitation was significantly shorter and the strength of the inhibition was stronger than those of the GPe. The inhibition was considered to be GABAergic, originating from the GPe, because the response was gabazine sensitive and an injection of the NBQX/CPP/gabazine mixture into the GPe blocked the response. Anatomical studies in both monkeys and rats have found no extensive local collaterals of GPi neurons (Nakanishi et al., 1991; Parent et al., 1999). STNBHFS evoked excitations similar to the GPe neurons in 25\% of the GPi neurons. However, in 75\% of the neurons, STN-BHFS evoked a short-latency excitation followed by inhibition. Local application of gabazine or injection of an NBQX/CPP/gabazine mixture into the GPe blocked the inhibition and disclosed the short-latency excitations. These observations suggested that STN-GPe-GPi inhibitions overrode monosynaptic STN-GPi excitation. To substantiate the results with electrical stimulation, we examined the effects of chemical activation of the STN on the spontaneous activity of pallidal neurons. Injection of gabazine into the STN increased the activity of all GPe neurons. In the GPi, 4 of 10 neurons were increased and 6 were decreased. These results were consistent with electrical stimulation results. Injection of gabazine into the STN occasionally resulted in dyskinetic movements of the contralateral forelimb and in a circling behavior. These observations agree with the previous study that injection of $\mathrm{GABA}_{\mathrm{A}}$ blocker, such as picrotoxin or bicuculline, into the STN evoked dyskinesia (Crossman, 1987), and consistent with the idea that blockade of information flow at the STN by muscimol or gabazine results in involuntary movements.

Previous studies reported that HFS of the STN inhibited activity of neurons in the $\mathrm{SNr}$, the nucleus that has similar inputs as the GPi. The inhibition was considered a result of the suppression of the STN itself or to the activation of GABAergic fibers in the STN (Benazzouz et al., 2000; Maurice et al., 2003). The results of the present study add another possibility that the STN-GPe-SNr inhibition may dominate over the STN-SNr excitation. The reports that HFS of the STN induced both glutamate and GABA release in the GPe and a larger GABA release in the $\mathrm{SNr}$ are consistent with our present observations (Savasta et al., 2002; Windels et al., 2003, 2005).

\section{Slow responses}

Single and BHFS of the STN evoked a slow excitation in GPe neurons. Local injection of the NBQX/CPP mixture diminished the slow excitation, suggesting that excitation of the GPe contributed to evoking the response. However, whether the response was caused by slow metabotropic responses or to loop connections involving other brain areas remains to be investigated. In the GPi, STN-BHFS was followed by a gabazine-resistant inhibition and slow excitations. Injection of an NBQX/CPP/gabazine mixture into the GPe also diminished these responses, suggesting a contribution of GPe excitation in inducing the responses.

\section{Responses of Put neurons}

The motor cortex and the centromedial nucleus of the thalamus project to both the STN and Put. Thus, it was possible that stimulation of the STN antidromically activated these structures and then the striatal projection neurons. Recording from PANs suggested that STN-BHFS could induce a slow excitation in some PANs, although single STN stimulation failed to excite PANs. The excitation to BHFS was weak and was considered not to be a significant factor in the responses observed in the pallidum in the present study. In contrast, a relatively large number of TANs responded with a weak excitation followed by inhibition and a second excitation to single STN stimulation, suggesting that STN stimulation indeed induced a short-latency excitatory drive in the Put. STN-BHFS failed to evoke strong responses in TANs, probably because the excitatory and inhibitory forces overlapped and canceled each other. These observations led to the possibility that the use of a STN stimulus electrode, which is more effective for activating cortical or thalamic afferent fibers than the electrode used in the present study, might induce significant excitations in the Put. The report that HFS of the STN increased striatal gluta- 
mate is consistent with present observations (Savasta et al., 2002). It can be further speculated that the excitations of striatal neurons may be beneficial for ameliorating parkinsonian symptoms.

\section{Functional considerations}

The most significant implication of this study is that STN-BHFS or STN-HFS might inhibit the activity of the majority of the neurons in the GPi, a basal ganglia output nucleus. STN neurons respond very strongly to task-related events or sensorimotor stimuli (Matsumura et al., 1992; Bergman et al., 1994; Wichmann et al., 1994). STN neurons in parkinsonian patients and experimental parkinsonian animals often show higher-frequency firing and increased burst activity (Bergman et al., 1994; Hutchison et al., 1998). The STN burst activity could be induced by both excitatory and inhibitory synaptic inputs and could be supported or enhanced by the reciprocal connections with the GPe and membrane properties of STN neurons (Bevan et al., 2002; Magill et al., 2004). The present results suggest that STN-BHFS would facilitate burst activity in the GPe by inducing monosynaptic excitations (Ni et al., 2000). However, the burst activity of STN activity may suppress activity or induce a pause followed by a rebound discharge in GPi neurons.

It has been suggested that overactivation of the indirect $\mathrm{SNr}$ GPe-STN-GPi pathway in the parkinsonian state increases the activity of the STN and then increases that of the GPi (Alexander and Crutcher, 1990; DeLong, 1990; Mink and Thach, 1993; Nini et al., 1995). This suggestion may not be so simple as long as the disynaptic STN-GPe-GPi projection is functioning normally. First, the increased STN activity might excite the GPe, acting as a negative feedback circuit, preventing a large decrease of GPe activity. Second, the elevated GPe activity effectively inhibits the GPi as well as the STN. Thus, the indirect pathway has build in feedback systems. It is possible to speculate from the present results that STN-HFS suppresses abnormally increased GPi activity in the parkinsonian states through the STN-GPe-GPi pathway, ameliorating parkinsonian symptoms. In contrast, STNBHFS or chemical activation of the STN evoked excitations in a substantial number of GPi neurons, agreeing with a report of an increased GPi activity during STN-HFS in parkinsonian monkeys (Hashimoto et al., 2003). Thus, an alternative explanation for the mechanism of the HFS treatment may be the disruption of the abnormal discharge patterns in the GPi in the parkinsonian state (Hashimoto et al., 2003; Maurice et al., 2003).

\section{References}

Alexander GE, Crutcher MD (1990) Functional architecture of basal ganglia circuits: neural substrates of parallel processing. Trends Neurosci 13:266-271.

Aziz TZ, Peggs D, Sambrook MA, Crossman AR (1991) Lesion of the subthalamic nucleus for the alleviation of 1-methyl-4-phenyl-1,2,3,6tetrahydropyridine (MPTP)-induced parkinsonism in the primate. Mov Disord 6:288-292.

Bar-Gad I, Elias S, Vaadia E, Bergman H (2004) Complex locking rather than complete cessation of neuronal activity in the globus pallidus of a 1-methyl-4-phenyl-1,2,3,6-tetrahydropyridine-treated primate in response to pallidal microstimulation. J Neurosci 24:7410-7419.

Barlas O, Hanagasi HA, Imer M, Sahin HA, Sencer S, Emre M (2001) Do unilateral ablative lesions of the subthalamic nucleu in parkinsonian patients lead to hemiballism? Mov Disord 16:306-310.

Benazzouz A, Gross C, Feger J, Boraud T, Bioulac B (1993) Reversal of rigidity and improvement in motor performance by subthalamic highfrequency stimulation in MPTP-treated monkeys. Eur J Neurosci 5:382-389.

Benazzouz A, Boraud T, Feger J, Burbaud P, Bioulac B, Gross C (1996) Alleviation of experimental hemiparkinsonism by high-frequency stimu- lation of the subthalamic nucleus in primates: a comparison with L-Dopa treatment. Mov Disord 11:627-632.

Benazzouz A, Piallat B, Ni ZG, Koudsie A, Pollak P, Benabid AL (2000) Implication of the subthalamic nucleus in the pathophysiology and pathogenesis of Parkinson's disease. Cell Transplant 9:215-221.

Bergman H, Wichmann T, DeLong MR (1990) Reversal of experimental parkinsonism by lesions of the subthalamic nucleus. Science 249:1436-1438.

Bergman H, Wichmann T, Karmon B, DeLong MR (1994) The primate subthalamic nucleus. II. Neuronal activity in the MPTP model of parkinsonism. J Neurophysiol 72:507-520.

Bevan MD, Magill PJ, Hallworth NE, Bolam JP, Wilson CJ (2002) Regulation of the timing and pattern of action potential generation in rat subthalamic neurons in vitro by GABA-A IPSPs. J Neurophysiol $87: 1348-1362$

Crossman AR (1987) Primate models of dyskinesia: the experimental approach to the study of basal ganglia-related involuntary movement disorders. Neuroscience 21:1-40.

Crutcher MD, DeLong MR (1984) Single cell studies of the primate putamen. I. Functional organization. Exp Brain Res 53:233-243.

DeLong MR (1971) Activity of pallidal neurons during movement. J Neurophysiol 34:414-427.

DeLong MR (1990) Primate models of movement disorders of basal ganglia origin. Trends Neurosci 13:281-285.

Dewey Jr B, Jankovic J (1989) Hemiballism-hemichorea. Clinical and pharmacologic findings in 21 patients. Arch Neurol 46:862-867.

Fujimoto K, Kita H (1993) Response characteristics of subthalamic neurons to the stimulation of the sensorimotor cortex in the rat. Brain Res 609:185-192.

Hanson JE, Jaeger D (2002) Short-term plasticity shapes the response to simulated normal and parkinsonian input patterns in the globus pallidus. J Neurosci 22:5164-5172.

Hashimoto T, Elder CM, Okun MS, Patrick SK, Vitek JL (2003) Stimulation of the subthalamic nucleus changes the firing pattern of pallidal neurons. J Neurosci 23:1916-1923.

Hutchison WD, Allan RJ, Opitz H, Levy R, Dostrovsky JO, Lang AE, Lozano AM (1998) Neurophysiological identification of the subthalamic nucleus in surgery for Parkinson's disease. Ann Neurol 44:622-628.

Kimura M, Rajkowski J, Evarts E (1984) Tonically discharging putamen neurons exhibit set-dependent responses. Proc Natl Acad Sci USA 81:4998-5001.

Kimura M, Kato M, Shimazaki H (1990) Physiological properties of projection neurons in the monkey striatum to the globus pallidus. Exp Brain Res 82:672-676.

Kita H (1994) Physiology of two disynaptic pathways from the sensorimotor cortex to the basal ganglia output nuclei. In: The basal ganglia IV. New ideas and data on structure and function (Percheron G, Mckenzie JS, Feger J, eds), pp 263-276. New York: Plenum.

Kita H, Kitai ST (1987) Efferent projections of the subthalamic nucleus in the rat: light and electron microscopic analysis with the PHA-L method. J Comp Neurol 260:435-452.

Kita H, Kitai ST (1991) Intracellular study of rat globus pallidus neurons: membrane properties and responses to neostriatal, subthalamic and nigral stimulation. Brain Res 564:296-305.

Kita H, Chang HT, Kitai ST (1983a) Pallidal inputs to subthalamus: intracellular analysis. Brain Res 264:255-265.

Kita H, Chang HT, Kitai ST (1983b) The morphology of intracellularly labeled rat subthalamic neurons: a light microscopic analysis. J Comp Neurol 215:245-257.

Kita H, Nambu A, Kaneda K, Tachibana Y, Takada M (2004) Role of ionotropic glutamatergic and GABAergic inputs on the firing activity of neurons in the external pallidum in awake monkeys. J Neurophysiol 92:3069-3084.

Kitai ST, Deniau JM (1981) Cortical inputs to the subthalamus: intracellular analysis. Brain Res 214:411-415.

Kitai ST, Kita H (1987) Anatomy and physiology of the subthalamic nucleus: a driving force of the basal ganglia. In: The basal ganglia II. Structure and function-current concepts (Carpenter MB, Jayaraman A, eds), pp 357-373. New York: Plenum.

Levy R, Hutchison WD, Lozano AM, Dostrovsky JO (2000) High-frequency synchronization of neuronal activity in the subthalamic nucleus of parkinsonian patients with limb tremor. J Neurosci 20:7766-7775. 
Lopiano L, Rizzone M, Bergamasco B, Tavella A, Torre E, Perozzo P, Valentini MC, Lanotte M (2001) Deep brain stimulation of the subthalamic nucleus: clinical effectiveness and safety. Neurology 56:552-554.

Magill PJ, Sharott A, Bolam JP, Brown P (2004) Brain state-dependency of coherent oscillatory activity in the cerebral cortex and basal ganglia of the rat. J Neurophysiol 92:2122-2136.

Matsumura M, Kojima J, Gardiner TW, Hikosaka O (1992) Visual and oculomotor functions of monkey subthalamic nucleus. J Neurophysiol 67:1615-1632.

Maurice N, Thierry AM, Glowinski J, Deniau JM (2003) Spontaneous and evoked activity of substantia nigra pars reticulata neurons during highfrequency stimulation of the subthalamic nucleus. J Neurosci 23:9929-9936.

McIntyre CC, Mori S, Sherman DL, Thakor NV, Vitek JL (2004) Electric field and stimulating influence generated by deep brain stimulation of the subthalamic nucleus. Clin Neurophysiol 115:589-595.

Mink JW, Thach WT (1993) Basal ganglia intrinsic circuits and their role in behavior. Curr Opin Neurobiol 3:950-957.

Nakanishi H, Kita H, Kitai ST (1991) Intracellular study of rat entopeduncular nucleus neurons in an in vitro slice preparation: response to subthalamic stimulation. Brain Res 549:285-291.

Nambu A (2004) A new dynamic model of the cortico-basal ganglia loop. Prog Brain Res 143:461-466.

Nambu A, Tokuno H, Hamada I, Kita H, Imanishi M, Akazawa T, Ikeuchi Y, Hasegawa N (2000) Excitatory cortical inputs to pallidal neurons via the subthalamic nucleus in the monkey. J Neurophysiol 84:289-300.

Ni Z, Bouali-Benazzouz R, Gao D, Benabid AL, Benazzouz A (2000) Changes in the firing pattern of globus pallidus neurons after the degeneration of nigrostriatal pathway are mediated by the subthalamic nucleus in the rat. Eur J Neurosci 12:4338-4344.

Nini A, Feingold A, Slovin H, Bergman H (1995) Neurons in the globus pallidus do not show correlated activity in the normal monkey, but phaselocked oscillations appear in the MPTP model of parkinsonism. J Neurophysiol 74:1800-1805.
Parent A, Hazrati LN (1995) Functional anatomy of the basal ganglia. II The place of subthalamic nucleus and external pallidum in basal ganglia circuitry. Brain Res Brain Res Rev 20:128-154.

Parent M, Levesque M, Parent A (1999) The pallidofugal projection system in primates: evidence for neurons branching ipsilaterally and contralaterally to the thalamus and brainstem. J Chem Neuroanat 16:153-165.

Sato F, Parent M, Levesque M, Parent A (2000) Axonal branching pattern of neurons of the subthalamic nucleus in primates. J Comp Neurol 424:142-152.

Savasta M, Windels F, Bruet N, Bertrand A, Poupard A (2002) Neurochemical modifications induced by high frequency stimulation of subthalamic nucleus in rats. In: The basal ganglia VII (Nicholsson L, ed), pp 581-590. New York: Kluwer Academic Plenum.

Shink E, Smith Y (1995) Differential synaptic innervation of neurons in the internal and external segments of the globus pallidus by the GABA- and glutamate-containing terminals in the squirrel monkey. J Comp Neurol 358:119-141.

Smith Y, Hazrati LN, Parent A (1990) Efferent projections of the subthalamic nucleus in the squirrel monkey as studied by the PHA-L anterograde tracing method. J Comp Neurol 294:306-323.

Wichmann T, Bergman H, DeLong MR (1994) The primate subthalamic nucleus. I. Functional properties in intact animals. J Neurophysiol 72:494-506.

Windels F, Bruet N, Poupard A, Feuerstein C, Bertrand A, Savasta M (2003) Influence of the frequency parameter on extracellular glutamate and gamma-aminobutyric acid in substantia nigra and globus pallidus during electrical stimulation of subthalamic nucleus in rats. J Neurosci Res 72:259-267.

Windels F, Carcenac C, Poupard A, Savasta M (2005) Pallidal origin of GABA release within the substantia nigra pars reticulata during highfrequency stimulation of the subthalamic nucleus. J Neurosci 25:50795086. 